\title{
Selection of tested component to predict dynamic properties of large structures by component modal tests
}

\author{
M. Misawa,a, J. Nakajima², M. Kobayashi, and H. Kawasoe \\ ${ }^{1}$ Shizuoka University, Department of Mechanical Engineering, 3-5-1 Johoku Hamamatsu, Japan \\ ${ }^{2}$ Advics, Technical Center Foundation Brake Engineering Department, 170-1 Yamanokami imagawa- \\ cho Kariya, Japan \\ ${ }^{3}$ Shizuoka University, Graduate student \\ ${ }^{4}$ All Nippon Airways, Haneda Airport Maintenance Line Maintenance Center, 1-5-2 \\ Higashishinbashi Minato-ku Tokyo, Japan
}

\section{Introduction}

Large flexible structures cause the full-scale ground tests to be impossible sometimes because they are not strong enough to withstand the force of gravity. The focus of the research into this problem is to confirm the dynamic characteristics of large structures without testing the whole structure. Admire et al. used the mass additive method in a modal test to avoid problems associated with design and development of test fixtures for Space Shuttle payloads [1]. Karpel et al. proposed a method to test components with boundary coordinates loaded with rigid, heavy dummy masses supported by soft springs [2]. Dummy masses generate local displacements near the boundaries to predict the dynamic properties of the whole structure. Misawa and Funamoto [3] proposed a method to predict the dynamic characteristics of large structures with component modal tests. In this method, to simulate the dynamic behavior of structures, reduced mass and stiffness matrices of untested components are replaced with a mass and a spring attached to a tested component. Misawa et al. showed a procedure to select a location with additional mass and stiffness for measuring accurate dynamic characteristics of structures by component modal tests [4]. This method needs no CMS.

\section{Proposed method}

This paper describes a method to predict the frequencies and modes of large structures by component modal tests. We consider large structures consisting of several elastic components and select a tested component subjected to modal tests. A tested component includes one or more components. The rest of the components are untested components. As the modal test of a tested component never gives the dynamic characteristics of structures, we consider the effect of untested components as additional mass and stiffness. They are found by reducing mass and stiffness matrices of a tested component and a structure to an arbitrary degree of freedom. In this study, additional mass and stiffness are attached to a translational coordinate on a tested component and they are attached to the same coordinate. After the completion of the modal test, a different tested component is selected. In this way, modal tests are performed for different tested components. We consider identical frequencies obtained by component modal tests as frequencies of structures.

a e-mail : tmmisaw@ipc.shizuoka.ac.jp

This is an Open Access article distributed under the terms of the Creative Commons Attribution-Noncommercial License 3.0, which permits unrestricted use, distribution, and reproduction in any noncommercial medium, provided the original work is properly cited. 


\subsection{Mass and stiffness additive location}

Using the dynamic equation of structures, we replace mass matrix $\boldsymbol{M}_{\mathrm{s}}$ of structures with a lumped mass (reduced mas $\bar{M}_{\mathrm{s}}$ ) attached to a mass additive coordinate. In a similar way, we obtain reduced stiffnes $\bar{K}_{\mathrm{s}}$ using the dynamic equation of the tested component. The difference between $\bar{M}_{\mathrm{s}}$ and $\bar{K}_{\mathrm{s}}\left(\bar{M}_{\mathrm{s}}\right.$ and $\left.\bar{K}_{\mathrm{s}}\right)$ denotes the effect of untested components and additional mass and additional stiffness are expressed by

$$
\left.\begin{array}{l}
\Delta M=\bar{M}_{s}-\bar{M}_{t} \\
\Delta K=\bar{K}_{s}-\bar{K}_{t}
\end{array}\right\}
$$

Reduced mass and stiffness $\left(\bar{M}_{\mathrm{s}}\right.$ and $\bar{K}_{\mathrm{s}}, \bar{M}_{\mathrm{t}}$ and $\left.\bar{K}_{\mathrm{t}}\right)$ increase at some frequencies, so we call these frequencies "peak frequencies". In this method, peak frequencies of two masses $\left(\bar{M}_{\mathrm{s}}\right.$ and $\left.\bar{M}_{\mathrm{t}}\right)$ give key information to select the optimal location. It is important to select a location where adds mass and stiffness to a tested component for accurate frequency measurements by component modal tests. The location is selected so that we separate peak frequencies of two masses from a target frequency

\subsection{Selection of tested component}

It is important which component we select as a location tested component for the measurement of the target mode. When we select unsuitable tested with additional mass components, it is difficult to obtain an accurate \& stiffness target mode even if we select mass and stiffness additive coordinates as mentioned above. We consider the second mode of a cantilever beam shown in Fig. 1 as an example.

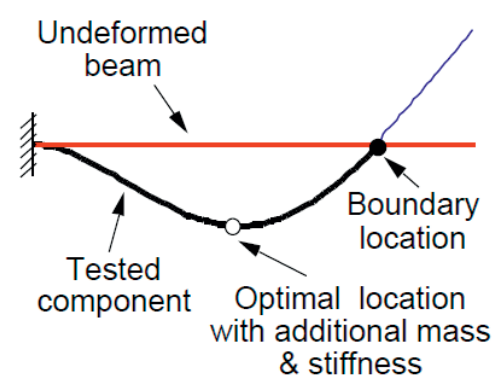

Fig. 1 Second mode of cantilever Because the boundary location of a tested component is free, displacement at the boundary is seldom small in component modal tests even though mass and stiffness are attached to the optimal location. We should add a large mass at the boundary to obtain the second mode with small boundary displacement. This means that it is impossible to obtain the target mode by procedure to select mass additive coordinates when the boundary of the tested component is close to the node of the target mode. Therefore, we need to select a tested component so that the boundary is separated from nodes of the target mode to measure accurate target modes of the structures with light additional mass. Because there is quite a possibility that a mass additive location changes for different tested components, we need to select mass additive location anew.

A numerical example is given to show that the proposed method has the potential to predict the dynamic characteristics of large structures by component modal tests.

\section{References}

1 Admire, J. R., Tinker, M. L., and Ivey, E. W., "Mass-Additive Modal Test Method for Verification of Constrained Structural Models," AIAA Journal, 31, 2148 (1993).

2 Karpel, M., and Raveh, D., "Fictitious Mass Element in Structural Dynamics," AIAA Journal, 34, 607 (1996).

3 Misawa, M., and Funamoto, K., "Dynamic Characteristic Prediction of Large Satellite Antennas by Component Tests," Journal of Spacecraft and Rockets, 42, 845 (2005).

4 Misawa M., Matsuo M., and Kojima S., "Component Modal Tests for Frequency Verification of Large Space Structures," Proceedings of the 26th International Modal Analysis Conference, Paper No. 20, 2008. 